\title{
Instalados en la queja: \\ El profesorado ante la participación de las familias en las escuelas
}

\section{Installed In The Grievance: Faculty Staff In Face Of The Families' Involvement In School}

\author{
Joaquín Giró Miranda y Sergio Andrés Cabello’
}

\begin{abstract}
Resumen
El profesorado es uno de los agentes de la comunidad educativa que más peso tiene en la participación de las familias en la escuela. Madres, padres y docentes son dos colectivos que están "condenados a entenderse" mientras la principal motivación e interés de ambos sea que sus hijos/alumnos consigan integrarse como ciudadanos, y adquieran el mayor rendimiento escolar. Tanto profesorado como familias cuentan con una amplia diversidad de actitudes, motivaciones e intereses que en ocasiones se muestran contrapuestos, lo que motiva el recelo del profesorado hacia la implicación de las familias. El presente artículo muestra ese recelo marcado por la desconfianza que lleva a establecer con frecuencia barreras y límites que no deberían cruzarse por parte de las madres y padres, principalmente en los aspectos académicos y en el aula, espacio que estaría consagrado a la función docente. Este artículo analiza desde el discurso del profesorado las actitudes de queja hacia la participación de las familias en la educación obligatoria, a través de una investigación de carácter cualitativo en la que han participado treintaiún centros de Educación Primaria y Secundaria.
\end{abstract}

\section{Palabras clave}

profesorado, participación, familias, escuelas.

\section{Abstract}

Teachers are one of the main actors in the educational community with regard to families' involvement in school. Parents and teachers are condemned to understand each other, while the main cause for concern is that children/pupils manage to integrate as citizens and gain greater educational achievement. Both teachers and families count on a wide variety of attitudes, motivation and interests which sometimes behave as competing concerns. This situation motivates the distrust of teachers towards families' involvement. This feeling of distrust is marked by the disbelief which leads to establish barriers and limits which shouldn't be crossed by parents. These limits refer to academic aspects and to the classroom, a space which must be devoted to teaching. This paper analyzes the complaints about families' involvement in compulsory education, starting from teacher's speech, through a research which involved thirty-one centers of Elementary and Secondary Education.

1 Profesor de Sociología, Universidad de La Rioja, joaquin.giro@unirioja.es; Profesor de Sociología, Universidad de La Rioja, sergio. andres@unirioja.es 
Key words

faculty staff, involvement, families, schools.

Recibido: 10-04-2016 Aceptado: 17-07-2016

\section{Introducción}

A la hora de analizar la participación de las familias en la escuela se debe tener en consideración a uno de los actores fundamentales, el profesorado. Partimos de que la participación se enmarca en el contexto de la comunidad educativa, un conjunto actoral donde participan estudiantes, madres y padres, docentes, equipos directivos, personal no docente y el entorno (Epstein, 2001; Fernández Enguita, 2007; Garreta, 2008; Garreta y Llevot, 2007; Giró et al., 2014). Entre todos ellos se producen relaciones y comunicaciones, estando entre las más importantes las que se dan entre familias y profesorado, hasta el punto de erigirse en protagonistas del concepto de participación en la escuela; sin embargo, son dos colectivos heterogéneos cuyas relaciones no son fáciles y entre los que se dan desconfianzas y recelos.

Los trabajos teóricos y empíricos sobre la participación en la escuela han abarcado un gran número de ámbitos posibles aunque es un proceso lejos de finalizar. Al contrario, a medida que nuestras sociedades se complejizan también lo hacen las familias, las escuelas y las relaciones entre ambas. A las segundas se les reclaman más funciones que antes asumían las instituciones familiares, pues si antes estaba clara la división de funciones ("la escuela enseña, la familia educa») boy la escuela está acumulando ambas funciones y -en determinados contextos- está obligada a asumir la formación en aspectos de socialización primaria (Bolívar, 2006: 121), hasta el punto de que los cambios en la sociedad pueden operar en otro sentido, sobrecargando el sistema escolar con nuevas y múltiples demandas, a las que las escuelas encuentran difícil responder. Según ello, se esperaría de los profesionales, individual o colectivamente, que desempeñaran funciones para las que tradicionalmente no estaban preparados (Pérez-Díaz y Rodríguez, 2012: 56). Efectivamente, esta representación sobre cuáles son las funciones de la escuela se ve sometida a cambios de sentido. Madres y padres también se relacionan de forma diferente con la escuela con una visión más cercana a la lógica del consumo, demandando servicios antes que ser copartícipes y corresponsables en el proceso educativo (Collet-Sabé, 2014; Symeou, 2005). Es un síntoma también de los cambios sociales, donde se perciben actitudes más individualistas y consumistas. Esta investigación corrobora que en los colegios e institutos se observa la preeminencia de la participación en las tutorías basada en los problemas que afectan a los hijos.

Tampoco todo el mundo entiende lo mismo por participar, estando claro que es una posición activa y que participar no es asistir, no es la mera asistencia numérica, ni el asentimiento o la oportunidad de responder a las propuestas de otros; no pasa siempre y necesariamente por la delegación en otros; no se limita al control indirecto de las decisiones y no es el ejercicio periódico del voto (De la Guardia, 2005: 134), sino que implica gestionar colectivamente la educación.

Así pues, este artículo se centrará en el profesorado, actor que desempeña un papel determinante en todo el proceso (Andrés y Giró, 2016). Y es que unos docentes que apuesten, faciliten y conciban que las familias son una parte fundamental de la educación, entendida globalmente, abrirán las puertas a madres y padres; en caso contrario, se pondrán barreras a las familias y las relaciones y comunicaciones serán más complejas. En consecuencia, nos encontramos con una diversidad de situaciones y escenarios, ya que el profesorado es un colectivo tan heterogéneo como el de las familias. Además, estas relaciones suelen 
venir determinadas en parte por las experiencias pasadas y, si han sido negativas, estos docentes no serán muy receptivos, como se ha podido observar en esta investigación. Sin embargo, la actitud del profesorado no solo va a depender de las biografías personales sino de otros factores, como la política de centro acerca de la participación, la titularidad del centro en el que se encuentran, la ubicación del centro, si es urbano o rural e incluso por las dimensiones del mismo, la edad y situación dentro de la carrera profesional, y la etapa educativa en la que imparte la docencia.

También, en el trabajo de campo hemos observado una serie de cuestiones comunes que aparecen asiduamente. Por un lado, la necesidad de participación de las familias en colaboración con el profesorado, ya que incide en el rendimiento escolar y académico (otra cosa es lo que cada uno entienda por la implicación de madres y padres), y por otro lado la necesidad de marcar unos límites y unas barreras entendidas como un espacio (el aula) y un ámbito (el académico) que se considera patrimonio de los docentes. Sin embargo, también habría que precisar la naturaleza y el punto de partida de esa participación, incidiendo en las características desiguales de las familias (especialmente las menos identificadas con la cultura de la escuela), así como la dimensión institucional de la escuela, y, cómo han probado numerosos estudios, puede generarse el efecto contrario, es decir, que perjudique el rendimiento de los estudiantes (Collet-Sabé et al., 2014; Pomerantz et al., 2007; Theodorou, 2007; Corter y Pelletier, 2005; Symeou, 2005). En todo caso, ambos actores buscan el mismo fin (el beneficio máximo de sus hijos/alumnos), aunque no dudan unos y otros en culpabilizarse si las relaciones y las comunicaciones entre ellos no funcionan y no son las deseadas cuantitativa o cualitativamente, demostrando una baja autocrítica. Finalmente, toda relación es un proceso bidireccional en el que es muy importante la retroalimentación, la actitud con la que se reciben los mensajes, todo lo cual condicionará las respuestas.

\section{Metodología}

Los resultados que se presentan en este artículo proceden del Proyecto de Investigación Familias y escuelas. Discursos y prácticas cotidianas sobre la participación en la educación obligatoria (EDU2012-32657) de la convocatoria 2012 del Subprograma de Proyectos de Investigación Fundamental no Orientada del Ministerio de Economía y Competitividad. Este estudio ha sido realizado por un equipo interdisciplinar (sociólogos de la educación, pedagogos, trabajadores sociales y psicólogos) que ha analizado experiencias de éxito en la participación de las familias en las escuelas en Cataluña, Aragón, Islas Baleares y La Rioja, en centros de características diversas como el perfil socioeconómico del alumnado que asiste a los mismos.

La metodología empleada ha sido de carácter cualitativo, llevándose a cabo etnografías en treinta y un centros de estas cuatro regiones durante la segunda mitad del curso 2013/14 y la primera del 2014/15. Se entrevistaron a equipos directivos, docentes, madres y padres, personal no docente y otros informantes cualificados, además de observar las dinámicas de participación, las relaciones que se daban en los centros, y las comunicaciones entre los diferentes agentes de la comunidad educativa, especialmente en colegios e institutos (equipos directivos y profesorado) y las familias (Asociaciones de Madres y Padres de Alumnos, y madres y padres). La muestra de centros tuvo en consideración las siguientes variables: etapa educativa (Centros de Educación Infantil y Primaria e Institutos de Educación Secundaria), titularidad de los centros (públicos y concertados), ubicación de los centros (urbanos y rurales), homogeneidad o diversidad del alumnado, así como también se realizaron etnografías en centros que se organizan como Comunidades de Aprendizaje.

En el caso de los docentes, durante el trabajo de campo se les entrevistó en función de sus diferentes niveles de enseñanza (Primero y Segundo de Primaria, Tercero y Cuarto de Primaria, Quinto y Sexto de Primaria, Primero y Segundo de Secundaria, y Tercero y Cuarto de Secundaria), así como entrevistas a 
equipos directivos formados por profesores. En su conjunto, por lo tanto, una muestra amplia y diversa, que cubre las etapas de Primaria y Secundaria, que nos ha permitido captar la heterogeneidad de este colectivo y sus motivaciones e inquietudes en relación a la participación de las familias en la escuela.

\section{El profesorado ante la participación de las familias}

No cabe duda de que el profesorado es uno de los actores claves de la participación de madres y padres en la escuela. El primer motivo es que los docentes son los interlocutores más directos y continuados con los que se van a encontrar las familias. Su relación con el equipo directivo es más puntual, por ejemplo, en las jornadas de puertas abiertas, en las reuniones de inicio de curso o trimestrales, o si hay alguna cuestión de consideración a tratar o que afecte a la disciplina y el comportamiento. Lo mismo ocurre con otros profesionales del centro o vinculados al mismo, como por ejemplo orientadores, personal de apoyo, etc., aunque las relaciones y comunicaciones cambian y disminuyen a medida que se avanza en las etapas educativas (ganando más peso el tutor con el discurrir de las mismas). Por lo tanto, la investigación llevada a cabo muestra que los docentes son en buena medida responsables de la implicación de las familias al ser ellos quienes abren o cierran esa puerta, y en la aplicación de una cultura de centro, aunque generalmente la dejan entreabierta. Esta es una visión que está completamente extendida entre todos los agentes de la comunidad educativa.

Las relaciones de los profesores con las familias es una de las preocupaciones más importantes. En el Barómetro del profesorado de 2010, el 46,3\% de los docentes encuestados señalaban que las relaciones con los padres eran un problema, situándose en la sexta posición de doce cuestiones en las que se valoraba si diferentes aspectos de la educación eran un problema. Más significativo resulta que en la puntuación asignada a la gravedad de distintos problemas del sistema educativo, la falta de apoyo de los padres alcanzase una valoración 8,26 puntos sobre diez, siendo el tercer problema de una lista de dieciocho y solo superado por el desinterés de los alumnos $(8,41)$ y por la crisis de valores en los alumnos $(8,44)$ (Fernández Enguita y Rivière, 2010). Así pues, la participación de las familias es considerada un problema funcional del profesorado.

Los docentes, además de ser un colectivo muy heterogéneo, también cuentan con una trayectoria. Hasta la década de los ochenta se puede observar una cierta senda unidireccional en familias y profesores centrada en la construcción de centros con la generalización de la enseñanza pública; a partir de esa década, y logradas las reivindicaciones profesionales de los segundos, esta senda se bifurca (Mata, 2014). Aunque es posible que en el contexto anterior a la década de los ochenta nos encontremos con unos límites más prefijados, y donde las visiones acerca de la comunidad educativa fuesen distintas a las actuales. Por otra parte, como bien reflejan diferentes estudios, dentro de la diversidad de actitudes que estructuran la relación con familias, no hay que olvidar que en las diferentes perspectivas de los docentes hay que tener en cuenta su adscripción ideológica, las teorías pedagógicas con que se manejan y el interés profesional con el que las aplican (Garreta, 2008).

A lo largo de nuestra investigación se ha constatado que los docentes son muy conscientes del valor de la participación, necesitan a madres y padres por diferentes motivos, desde el mayor conocimiento de los alumnos y las situaciones que les afectan, hasta el generar una visión positiva del centro y de la educación que facilite una identificación con la misma, corroborando que cuando las interacciones entre la escuela y la familia se multiplican y existe un conocimiento mutuo del contexto en el que transcurre dicho aprendiraje, pueden identificarse con mayor precisión las barreras que dificultan el rendimiento académico infantil (Pérez-Díaz et al., 2009: 101). Si las familias cuestionan al docente, a la institución, se produce de cara al estudiante una deslegitimación de los primeros y el proceso educativo se torna más complejo. 
Pero la participación para los docentes puede ser una cosa y para las familias otra, y en realidad es así. La visión de la participación de los profesores también es muy heterogénea, y no podía ser de otra manera, pues la gran mayoría de los docentes entrevistados, en general tienen un punto en común muy claro, pues todos afirman que familias, escuelas y docentes tienen que ir en la misma dirección, la delineada desde el centro y el profesorado. Es decir, nos revelan que la relación es asimétrica, y donde los límites pedagógicos y académicos están claramente definidos y verbalizados en sus discursos.

Limites, tenemos que tener, porque si no estariamos aqui todo el día con ellos, en este caso, en este centro, es que los limites sabemos hasta dónde podemos llegar. (CPUFP2LR14AI).

Si que es verdad que, a veces, los padres, o depende de quépadres, consideran que su presencia y su participación en el colegio es decidir, y ahi están equivocados. Su labor no es la de decidir, su labor es la de apoyar, comentar, la de sugerir, la de informar, ese tipo de cuestiones, pero el verbo conjugar lo tiene que bacer la institución. (CIPCDLR14A).

Incluso algunos profesores llegarían a incorporar, sustituir, participación por coordinación con todas las connotaciones que la acompañan. En definitiva, los roles están definidos y marcados, institucionalizados, y todos los agentes deben tener claro cuál es su papel y espacio.

Desde luego si yo soy buen docente con mis alumnos, me encanta también serlo con los padres y explicarlo, pero a veces hay problemas abi de límite... tenemos que saber qué es la parcela de cada uno. (CPUPP1AR14AIII).

En cierto sentido y en un nivel más amplio, sería lo que De la Guardia definiría como psesudoparticipación";, no contando para la toma de decisiones y centrándose en que la participación de padres, profesores y alumnos pasa porque estén informados, debatan y aporten sugerencias al centro (2005: 138).

En esta investigación hemos observado la existencia de diferentes visiones del profesorado acerca de la participación de las familias en función de diversas variables. Una de las más claras es la relacionada con la etapa educativa en la que se inserta. En Infantil, la participación de madres y padres es buscada y demandada por los docentes, y la complicidad de los primeros es total ya que hay una visión más sobreprotectora con respecto a los niños. En Primaria se produce una ruptura en esa implicación que se hace más explícita cuando se alcanza el tercer ciclo, aunque en la primera etapa de la educación obligatoria el papel de las familias se centra en gran medida en el apoyo a los deberes. Y la brecha se hace definitiva en Secundaria, cuando en los centros públicos se produce el paso de los Centros de Educación Infantil y Primaria (CEIP) a los Institutos de Educación Secundaria (IES), donde la relación es todavía más individualizada y se centra en los resultados académicos y en cuestiones de comportamiento o disciplina, cobrando especial relevancia la figura del tutor como interlocutor en la relación familia-centro. El paso de la Primaria a la Secundaria también influye en que exista un mayor distanciamiento de las familias, el deseo de autonomía de los hijos, así como por el contenido de las materias y la complejidad de las decisiones a tomar por los estudiantes, que daría lugar a unos padres más inclinados a confiar en el juicio de sus hijos (Pérez-Díaz et al., 2009). En el caso de los concertados, que abarcan en su mayoría desde Educación Infantil hasta Secundaria, se observa en el trabajo desarrollado el mismo descenso de la participación con el paso de las etapas, pese a darse un mayor sentimiento de comunidad.

La cercanía con el profesorado en el medio rural tiene una parte positiva como es la transparencia y accesibilidad, pero también una cara negativa relativa a un "sentirse vigilado". En un contexto donde priman las relaciones informales, la rotación del profesorado dificulta esta participación. Son muchos los docentes que cambian en estos centros al finalizar el curso, lo que implica que las relaciones de confianza 
se tienen que recomponer cada año y que las visiones sobre la participación puedan ser diferentes. Además, muchos de estos profesores tienen su mirada en un objetivo profesional que es conseguir una plaza en la localidad de origen, lo que también puede condicionar esta visión sobre la participación.

Con respecto a los centros concertados, aunque se apunta constantemente hacia el concepto de comunidad o identidad, no es menos cierto que las relaciones familia-escuela estarían marcadas por ciertos límites que en parte son herencia de estructuras del pasado más jerarquizadas. Aunque son colegios que poseen AMPAs potentes, una visión muy extendida es la de contar con las familias como apoyo al centro. Se les consulta, pero dentro de los cauces que señala el propio centro. No es un proceso exclusivo de los concertados ya que también se aprecia en parte de los públicos, como se ha constado en el trabajo de campo llevado a cabo.

Donde se trata de superar estas barreras es en las Comunidades de Aprendizaje, modelo que hace gala de la participación como parte de su identidad y mecanismo de funcionamiento (Elboj y Oliver, 2003). Abiertos a las familias y al entorno a través de herramientas como las comisiones, las tertulias dialógicas, los grupos interactivos, etc., el proceso sería colaborativo al incluir los ámbitos de gestión y pedagógicos. Pero, en general, se ha observado que las Comunidades de Aprendizaje también cuentan con buena parte de los problemas comunes al resto de centros como la diversidad de las visiones del profesorado acerca de la participación y la poca implicación del conjunto de las familias, aunque las que lo hacen son muy activas.

En cualquier caso, son fundamentales los canales de comunicación entre familias y docentes, y se trata de mantenerlos siempre abiertos y que no se produzcan ruidos o disonancias entre ellos, hecho corroborado en todas las etnografías. Tutorías individuales y colectivas, notas, llamadas telefónicas, correos electrónicos, la agenda escolar, etc., son los empleados (Garreta y Llevot, 2015 y Llevot y Bernard, 2015), aunque también hay sitio para canales informales (en el patio, a la entrada y la salida) y crece el uso de Internet y las plataformas que permiten un contacto más continuado (Macià, 2016). Un mal uso por exceso o por defecto de la comunicación repercute también en las relaciones entre familias y docentes.

En general, a las familias, en no pocas ocasiones se las busca para actividades de carácter lúdico y festivo, que también es una forma de participación, pero no para cuestiones que tienen que ver con el ámbito pedagógico o, en un límite superior, con la gestión del centro. En este sentido, y dentro del esquema de la participación de las familias basada en las variables formal/informal e individual/colectiva, la tutoría individual sigue siendo el principal medio de participación y comunicación, y los docentes lo asumen y lo tienen interiorizado (Giró et al., 2014).

Pero sin duda alguna, uno de los principales aspectos que aparecen en todas las etnografías y entrevistas realizadas es la falta de formación para la participación, lo cual afecta al conjunto de la comunidad educativa. Este hecho ya se refleja en estudios nacionales e internacionales (Collet-Sabé et al., 2014; Pelletier y Corter, 2005; Symeou, 2005). En general, no hay una gran participación de carácter cívico y social, y en la escuela los docentes tampoco cuentan con una formación específica para relacionarse con las familias y fomentar esa implicación. De esta forma, nuestros interlocutores señalaban la inexistencia de una preparación, exceptuando el caso específico de las Comunidades de Aprendizaje, aunque también insuficiente, pero parte de ellos ponían el acento en las familias. Ni madres ni padres estarían preparados para la participación, pero tampoco los profesores, siendo una materia que no está presente o de forma muy parcial, en los planes de estudio de los Grados de Educación Infantil y Primaria y en el Máster de Secundaria. 


\section{Quejas, límites y barreras del profesorado}

En este epígrafe se desarrollan cuestiones como la visión dicotómica acerca de la participación de las familias, la necesidad de la misma y el lamento por su falta de implicación a los límites fijados, así como el temor a ser evaluados o juzgados. No debemos tampoco dejar de lado otras cuestiones de carácter contextual que afectan al profesorado en particular y a la educación y la sociedad en general, y que tiene una importante incidencia en la visión de la participación de las familias y en cómo el profesorado responde ante la misma.

En ese contexto más general destaca la visión que los docentes tienen de su papel en la sociedad y del que ocupa la Educación. Partiendo de que el sistema educativo, incluido el profesorado, ha tenido que asumir más funciones debido a las transformaciones aceleradas en las que se encuentra la sociedad y las familias, los docentes lamentan el poco valor que éstas le dan a la Educación y a la profesión. De hecho, en el ya mencionado Barómetro del profesorado, los docentes encuestados calificaban el reconocimiento socialcon 4,03 puntos sobre diez en una serie de trece características del empleo. Además de ocupar la última posición, era el único aspecto que suspendía junto a las oportunidades de carrera con 4,58 puntos (Fernández Enguita y Rivière, 2010).

Este es un dato erróneo a priori ya que la sociedad les tiene en alta estima como manifiestan las diferentes encuestas realizadas (Pérez-Díaz y Rodríguez, 2012). Otra cuestión son las relaciones cuando se llevan al terreno interpersonal y a las experiencias subjetivas. Hay profesores entre los entrevistados que lamentan la pérdida de una cierta autoridad, del respeto que tenían en el pasado y que madres y padres cuestionen su labor, cosa que no ocurriría con otras profesiones, como los médicos

\section{Habría que ponerlo, lógicamente, es igual que cuando... de Educación todo el mundo opina y todo el mundo sabe. Hay que marcar un límite porque el profesional es el que está dentro de la escuela, somos profesionales y bay defenderlo. De la Educación, todo el mundo sabe y todo el mundo opina. (CPUOPLR14AI).}

Sin embargo, la mayoría de los docentes asume que este proceso es imparable, que las relaciones ya no se pueden basar en ese rol de autoridad tan definido, y que el nivel formativo y cultural de madres y padres ha crecido en las dos últimas décadas, demandando relaciones de igual a igual y sin complejos, a diferencia de las generaciones de sus padres (Usategui y Del Valle, 2009: 24).

Esa intromisión de las familias va a más en el colegio donde las familias tienen más nivel que en los colegios donde las familias tienen menos nivel. Aqui puede haber familias más reivindicativas, que demanden mucho, y eso nosotros lo vemos como una intromisión en nuestro trabajo. (CPUDLR14AII).

Ahora bay padres que tienen un nivel educativo superior a losprofesores. He escuchado comentarios de 'cómo habla, cómo escribe'. Claro, es que igual estábamos acostumbrados a unos padres que tenían un nivel educativo... pero es que ahora hay padres universitarios, abogados, economistas. (IPUPS2LR14AII).

En ese sentido, algunos autores apuntan que estaríamos ante una transformación de un tipo de autoridad difusa, o que directamente ha desaparecido, a una más específica en una sociedad más diferenciada (García, 2012: 22).

Las familias también están en un proceso de transformación constante. Los nuevos modelos familiares, los cambios de roles en el seno de la institución, la incorporación de la mujer al mercado de trabajo, etc., han incidido en ese traspaso de funciones socializadoras desde la familia a la escuela. Tener que afrontar este proceso para el que no están preparados, también genera mucha inquietud y frustración en los docentes (Usategui y Del Valle, 2009), y no hay que olvidar que en un mundo más complejo y rápido, 
parte de las familias puede que no tengan la Educación de sus hijos como una de sus prioridades, descargando esa responsabilidad en escuela y profesores, reclamándoles resultados como indican algunos entrevistados.

En este sentido hay que hacer hincapié en un proceso global que afecta al sistema educativo, como es la relación clientelar que establecen parte de las familias con el mismo. En un modelo de sociedad basado en estructuras de consumo, hay madres y padres que establecen esa lógica con la escuela, demandándole servicios y resultados y depositando la responsabilidad de los mismos exclusivamente en la institución escolar. Esta visión de la relación colocaría a las familias en una posición de superioridad con respecto a docentes y centros, y se verían con la legitimidad de demandar, reclamar, criticar, cuestionar, etc.; y aunque no es una posición mayoritaria, sí que es creciente y perturba a los profesores, porque en este contexto no hay forma posible de remar en la misma dirección, como observan buena parte de los docentes entrevistados. La escuela y la Educación también son copartícipes de este proceso al incidir en discursos basados en la calidad, entendida ésta no como un derecho sino como un elemento de diferenciación, hecho que también hemos podido observar en esta investigación que también abordan otros autores (Collet-Sabé et al., 2014; Bolívar, 2006). Se produciría así una situación contradictoria, en la que las familias se moverían entre la responsabilidad y el clientelismo (Rivas, et al., 2011: 166).

Otro aspecto contextual que determina las quejas de los profesores en su relación con las familias hace referencia a las consecuencias de la crisis que comenzó en 2008. La Educación ha sido uno de los ámbitos más afectados por las políticas de recortes, a lo que se suma el rechazo de la LOMCE, finalmente aprobada a finales de 2013. No cabe duda de que este contexto no favorece el desempeño de la labor docente y afecta a las relaciones con las familias, en tanto los profesores tienen que asumir más funciones con menos recursos y en una situación laboral más precaria, siendo una situación verbalizada recurrentemente en el trabajo de campo. Pero este escenario también chocaría con la incomprensión que señalan los docentes de una parte de la sociedad, dentro de la queja sobre su valoración global, siendo vistos como privilegiados por su estabilidad en el empleo, sus salarios, sus vacaciones, etc., y generando más desconfianza entre familias y profesorado.

Hemos señalado la importancia de la formación, tanto de las familias como del profesorado, y cómo los segundos achacaban a las primeras ese déficit, una de las quejas más frecuentes entre docentes en el sentido de que las familias no estarían preparadas para la participación, traspasarían los límites que les tienen asignados y no sabrían relacionarse con los profesores. Se trasluce en este discurso esa visión asimétrica de la participación, en el sentido de que son ellos los que marcan la dirección a seguir. Y la situación se complejiza en dos caminos, por uno la creciente diversidad de las familias, incluida la cultural, que llevaría a algunos colectivos a contar incluso con barreras de comunicación derivadas del idioma (Garreta, 2009); y por otro, los cambios en la relación de las familias con la escuela, con unas familias que cada vez adoptan un papel más clientelar y no colaborativo, que demandan más funciones a los centros y docentes, y que además pueden igualar e incluso superar el nivel formativo de los profesores. No hay que negar que se producen situaciones en las que las familias traspasan los límites básicos de cualquier relación y marcan futuras interacciones, de modo que la desconfianza presente entre los dos colectivos se fortalece y se hace más difícil de superar.

Según el profesorado, también cabe reseñar la falta de empatía de las familias en su relación con los docentes. La incomprensión de la que harían gala madres y padres, incluso deslegitimando el papel de los profesores en el caso de conflictos con los estudiantes o culpabilizándolos directamente de los malos resultados académicos de sus hijos/as, pesa como una losa en la visión que los docentes tienen del papel de 
la participación, contribuyendo a marcar esos límites y barreras, factor que se corrobora en otros estudios de carácter cuantitativo (García-Bacete, 2006).

Otro aspecto significativo es el lenguaje empleado por los profesores al describir sus relaciones con las familias. En este sentido, y teniendo en cuenta la performatividad del mismo, son frecuentes las referencias a conceptos como "intromisión", "invasión", "confrontación”, "enemigos", "defensa”, etc., en su relación con madres y padres. Es una visión que lastra de entrada las relaciones y comunicaciones, pero también hay que indicar que en buena medida viene marcada por las relaciones anteriores y por experiencias negativas (Gomila y Pascual, 2015).

Creo que bay una cierta prevención, una cierta actitud defensiva, que los padres se queden en su parcela, no en una parcela que no les corresponde, que vengan aqui, que escuchen lo que les decimos, que no pretendan saber más que yo en ciertas cosas o que no tengan más capacidad de decisión en el centro. (IPUPS2LR14AII).

En todo caso, los límites y los ámbitos de cada uno de los agentes están prefijados. Los docentes destacan que son necesarios y están claramente establecidos en los aspectos académicos, pedagógicos y en el aula; incluso el profesorado cuando se parte, como regla inviolable, de que nadie cuestione ni se «meta» en su trabajo, y cualquier intervención de las familias se toma como una agresión. Este profesionalismo imposibilita, de partida, la colaboración (Bolívar, 2006: 132). En un nivel superior estaría la gestión, donde la visión más general es también contraria a la participación de las familias. No se indica que no se informe o no haya transparencia para generar climas de confianza, pero cada uno debe entender cuál es su rol en la comunidad educativa.

Hay otras cuestiones que también son recurrentes en las quejas de los docentes, como por ejemplo la falta de disponibilidad y de tiempo de las familias para ser atendidas o recibidas y que algunos estudios han señalado como uno de los factores más importantes que dificultan la relación con las familias. En nuestra investigación se ha comprobado que esta situación suele ser resuelta a costa de los profesores que articulan horas de tutorías para estar con madres y padres fuera del horario estipulado. En este sentido se observa una buena disposición de los docentes que parte de las familias no valoran.

Y también se señala con frecuencia el cansancio o el hartazgo de una parte de los profesores en la reclamación de la participación de las familias y en su escasa respuesta. Esto se da en situaciones de carácter colectivo, como por ejemplo reuniones trimestrales (que en algunos IES incluso se han llegado a suspender), y en llamadas y notificaciones no atendidas, especialmente en el caso de estudiantes procedentes de familias desfavorecidas que presentan problemas de comportamiento y disciplina, o que sus resultados son negativos, así como en aquellas que se encuentran más alejadas del modelo de escuela existente (Río, 2010). Los docentes también se cansan de reclamar la participación, que muchas veces va a depender de la existencia de un colectivo, o de un grupo de familias implicado y activo.

Muy interesante resulta la valoración de las relaciones y las comunicaciones informales, que situadas fuera de los mecanismos institucionalizados y produciéndose en las entradas o salidas del centro, en encuentros en pasillos o patios, etc., cristaliza en una visión dicotómica de las mismas. Por una parte se perciben como positivas al fortalecer la relación y clima de confianza y, por otra, se consideran un medio para superar los límites formales. Y aquí no encontramos consenso entre los docentes entrevistados, porque mientras que para unos son positivas y necesarias, las potencian y fomentan, para otros son negativas, las evitan y critican ese acercamiento de las familias.

Esto nos lleva a considerar algo que no hay que olvidar, y es que los docentes no dejan de ser personas, no solo con sus trayectorias, biografías y con el impacto que el contexto pueda tener en ellos, sino con sus miedos, inseguridades, etc. De esta forma, el temor a ser evaluado, juzgado o cuestionado es legítimo, 
especialmente en un contexto que da cada vez más pie para ello, propio de una relación clientelar. Pero también habría que preguntarse hasta qué punto este contexto no viene marcado por la naturaleza de una institución como la escolar, en la que los profesores no suelen visitar las aulas de sus compañeros y en el que cada uno se limita a un espacio y ámbito determinado.

\section{Conclusiones}

El discurso de la participación de las familias por parte del profesorado muestra, aparentemente, un mensaje contradictorio, pues por un lado se da una exigencia y un deseo de la implicación de madres y padres y, por otro, se manifiesta contrario a la misma si se sienten invadidos en lo que consideran sus ámbitos y espacios propios y prácticamente exclusivos.

Así pues, hablemos de centros públicos o de centros concertados, hablemos de profesorado de Primaria o de Secundaria, o hablemos de centros innovadores en materia de participación, existe un discurso ambivalente que opone a profesorado y familias. Unos y otros se pasan la responsabilidad del fracaso en sus relaciones mediante un discurso funcional que delimita los roles de ambos.

En todo caso, parece claro que el profesorado es consciente de su posición en esta relación asimétrica, en la que ellos serían los que deben marcar la dirección pidiendo a las familias su colaboración para seguir ésta. Los docentes asumen la necesidad de la participación pero se sienten desbordados y sin herramientas para afrontarla en un contexto cambiante y complejo como el actual, donde las familias transforman su relación con la escuela. En este sentido, el lamento por la perspectiva que tienen acerca de cómo les valora la sociedad, funcionaría como una justificación para esa desconfianza hacia las familias, apuntalada por experiencias negativas en las que se sintieron señalados y cuestionados.

El papel de la formación se antoja definitivo para superar este escenario de desconfianza, porque solo a través de ella se podrían articular relaciones basadas en la empatía y en la confianza. Aunque en sus discursos hacen referencia constantemente a la transparencia y al mantener informados a las madres y padres, cuestión que se facilita e incrementa con las Tecnologías de la Información y el Conocimiento (González-Patiño y Poveda, 2015; Macià, 2016), esta situación no hace sino generar tensiones adentrándose en un círculo vicioso donde la información se convierte en un fin y no en un medio. Encontrar nuevos espacios de colaboración a través de la formación, comenzando por los propios planes de estudios y siguiendo por la indispensable formación continua, es imprescindible ya que el profesorado junto con los centros y los equipos directivos, tienen que fomentar, incitar y facilitar la participación de las familias. De hecho, la necesidad de formación viene planteándose en casi todos los estudios y trabajos que abordan esta cuestión (De la Guardia, 2005; Santos, 2010), responsabilizando a la administración educativa de su poca insistencia en el funcionamiento y la formación de los distintos órganos e individuos (García-Bacete, 2006: 248).

Finalmente, dentro de esa visión dicotómica del profesorado acerca de la participación de las familias, cabría preguntarse si no hay también un discurso políticamente correcto sobre la misma, que luego no encuentra su correlato en las quejas y límites señalados. Ciertamente los docentes no pueden escapar de la participación, cuando valoran como muy satisfactorias las experiencias positivas pese a aprender sobre la marcha y sin una dirección o un plan prefijado mientras permanece la sombra de la desconfianza hacia las familias. 


\section{Referencias bibliográficas}

Andrés, S. y Giró, J. (2016): El papel y la representación del profesorado en la participación de las familias en la escuela, Revista Electrónica Interuniversitaria de Formación del Profesorado, 19 (1) 61-71. DOI: dx.doi. org/10.6018/reifop.19.1.245461

Bolívar, A. (2006): Familia y escuela: dos mundos llamados a trabajar en común, Revista de Educación, 339 97-118.

Collet-Sabé, J.; Besalú, X.; Feu, J. y Tort, A. (2014): Escuelas, familias y resultados académicos. Un nuevo modelo de análisis de las relaciones entre docentes y progenitores para el éxito de todo el alumnado. Profesorado, Revista de curriculum y formación del profesorado, 18 (2) 7-33.

Corter, C. y Pelletier, J. (2005): Parent and Community Involvement in Schools: Policy Panacea or Pandemic?, en: N. Bascia, A. Cumming, A. Datnow, K. Leithwood y D. Livingstone (eds.), International bandbook of educational policy (Dordrecht the Netherlands, Kluwer Press).

De la Guardia, R. M. (2005): Las concepciones del profesorado y padres sobre la participación educativa y su relación con la implicación de las familias, PSICNOSIS, Revista de Evaluación e Intervención Socioeducativa, 10 133-145.

Elboj, C. y Oliver, E. (2003): Las comunidades de aprendizaje: Un modelo de educación dialógica en la sociedad el conocimiento, Revista Interuniversitaria de Formación del Profesorado, 17 (3) 91-103.

Epstein, J. (2001): School, Family and Community Partnerships. Preparing Educators and Improving Schools (Boulder, Westview Press).

Fernández Enguita, M. y Rivière, J. (2010): Barómetro del profesorado, Escuela, 3.869 23-26.

Fernández Enguita, M. (2007): Educar es cosa de todos: escuela, familia y comunidad, en: J. Garreta (ed.) La relación familia-escuela (Lleida, Ediciones Universidad de Lleida).

García, P. (2012): Contexto del Informe: el debate sobre el prestigio de la profesión docente, en: V. Pérez-Díaz y J. C. Rodríguez (dirs.) El prestigio de la profesión docente en España. Percepción y realidad (Madrid, Fundación Europea Sociedad y Educación y Fundación Botín).

García-Bacete, F. J. (2006): Cómo son y cómo podrían ser las relaciones entre escuelas y familias en opinión del profesorado, Cultura y Educación, 18 (3-4) 247-265.

Garreta, J. (2009): Escuela y familias inmigradas: relaciones complejas, Revista Complutense de Educación, 20 (2) 275-291.

Garreta, J. (2008): La participación de las familias en la escuela pública. Las asociaciones de madres y padres del alumnado. (Madrid, Editores Centro de Investigación y Documentación Educativa y Confederación Española de Asociaciones de Padres y Madres de Alumnos).

Garreta, J. y Llevot, N. (2015): Family-School Communication in Spain: Channels and their Use, Ehquidad. International Welfare Policies and Social Work Journal, 3 29-48. DOI: 10.15257/ehquidad.2015.0002

Garreta, J. y Llevot, N. (2007): La relación familia-escuela: ¿una cuestión pendiente?, en: J. Garreta (ed.) La relación familia-escuela (Lleida, Ediciones Universidad de Lleida).

Giró, J.; Mata, A.; Vallespir, J. y Vigo, B. (2014): Familias y escuelas: los diferentes discursos sobre la participación, Ehquidad. International Welfare Policies and Social Work Journal, 2, 65-90. DOI: 10.15257/ ehquidad.2014.0009 
Gomila, M. A. y Pascual, B. (2015): La participación de las familias en el sistema educativo: la percepción del profesorado en formación, Revista Electrónica Interuniversitaria de Formación del Profesorado, 18 (3) 99 112. DOI: $10.6018 /$ reifop.18.3.199321

González-Patiño, J. y Póveda, D. (2015): Privileging the Individual Through the Collective Commitment: Parental Strategies and Dynamics of Involvement in a Middle Class School, Multidisciplinary, Journal of Educational Research, 5 (3)316-336. DOI: 10.17583/remie.2015.1524

Llevot, N. y Bernard, O. (2015): La participación de las familias en la escuela: factores clave, RASE. Revista de la Asociación de Sociología de la Educación, 8 (1) 57-70. Disponible en http:/ /www.ase.es/rase/index. $\mathrm{php/RASE/article/view/370}$

Macià, M. (2016): La comunicación familia-escuela: el uso de las TIC en los centros de Primaria, Revista Electrónica Interuniversitaria de Formación del Profesorado, 19 (1) 73-83. DOI: 10.6018/reifop.19.1.245841

Mata, A. (2014): Evolución de la participación de las familias en el sistema escolar: de la ilusión al desengaño. En Tiempos críticos en educación, XVII Conferencia de Sociología de la Educación, Bilbao, 8, 9 y 10 de julio de 2014.

Pelletier, J. y Corter, C. (2005): Toronto First Duty: Integrating Kindergarten, Childcare and Parenting Support to Help Diverse Families Connet to Schools, Multicultural Education, 13 (2) 30-37.

Pereda, V. (2006): La participación de las familias en los centros educativos. Algunos pasos dados. Mucho camino por recorrer, en López (ed.), La familia en el proceso educativo (Madrid, Ediciones Cinca).

Pérez-Díaz, V. y Rodríguez, J. C. (2012): Educación y prestigio docente en España: la visión de la sociedad, en: V. Pérez-Díaz y J. C. Rodríguez (dirs.) El prestigio de la profesión docente en España. Percepción y realidad (Madrid, Fundación Europea Sociedad y Educación y Fundación Botín).

Pérez-Díaz, V., Rodríguez, J. C. y Fernández, J. J. (2009): Educación y familia. Los padres ante la educación general de sus hijos en España (Madrid, Fundación de las Cajas de Ahorros).

Poomerantz, E. M.; Moorman, E. A. y Litwack, S. D. (2007): The How, Whom and Why of Parent's Involvement in Children's Academic Lives: More is Not Always Better, Review of Educational Research, 77 (3) $373-410$

Río, M. (2010): No quieren, no saben, no pueden: categorizaciones sobre las familias más alejadas del campo escolar, Revista Española de Sociología, 14 85-105.

Rivas, J. I.; Leite, A. y Cortés, P. (2011): Paradojas y conflictos entre las culturas del profesorado, las familias y los estudiantes en el contexto escolar, Revista de Educación, 356 161-183.

Santos M. (2010): La formación del profesorado en las instituciones que aprenden, Revista Interuniversitaria de Formación del Profesorado, 68 (24, 2) 175-200.

Symeou, L. (2005): Past and Present in the Notion of School-Family Collaboration, Aula Abierta, 85 165-184.

Theodorou, E. (2007): Reading Between the Lines: Exploring the Assumptions and Implications of Parental Involvement, International Journal About Parents in Education, 1 (0) 90-96.

Usategui, E. y Del Valle, A. I. (2009): Luces y sombras en la función docente desde la mirada del profesorado, REIFOP, 12 (2)19-37. Disponible en http://www.aufop.com/aufop/uploaded_files/articulos/1248477521.pdf 